\title{
Dissipation by identical oscillators
}

\author{
Michael Wilkinson $\dagger$ \\ Nuclear Science Division, Lawrence Berkeley Laboratory, Berkeley, CA 94720, USA
}

Received 19 February 1990

\begin{abstract}
This paper considers a microscopic model for dissipation, in which a degree of freedom $X$ is coupled to a large number of identical microscopic oscillators (typically non-interacting fermions in a potential well). The motion of the coordinate $X$ causes irreversible excitation of the gas of oscillators, which can be observed as a dissipation of the motion of this coordinate. The rate of dissipation depends drastically on whether the classical motion of the oscillators is regular or ergodic. A possible experimental demonstration of this effect is discussed.
\end{abstract}

\section{Introduction}

The mechanism of dissipation, the irreversible conversion of work into heat, is always the transfer of energy from a small number of observed coordinates into a large number of unobserved microscopic degrees of freedom. Often the microscopic degrees of freedom are modelled by a bath of dissimilar harmonic oscillators, with a continuous distribution of frequencies (see, e.g., Caldeira and Leggett 1981); this model is appropriate for many systems in which the microscopic degrees of freedom are bosons. In this paper, a different model is considered: the microscopic degrees of freedom are identical and independent, but can have arbitrary dynamics. In both of the applications discussed below, these degrees of freedom are weakly interacting fermions confined in a potential well. The independent-particle picture is natural for fermion systems at low temperatures, because the low-energy excitations can be modelled as independent quasiparticles (Pines and Nozieres 1966). The principal result of this paper is to point out that in this model the rate of dissipation is drastically reduced if the motion of the microscopic oscillators is regular, compared with similar systems exhibiting chaotic motion. The possibility of observing this effect in the absorption of radiation by small conducting particles is discussed.

The observable degree of freedom will be denoted by $X$, and it appears as a parameter in a classical Hamiltonian $H(\boldsymbol{q}, \boldsymbol{p} ; X)$ describing the microscopic degrees of freedom. It will be assumed that the rate of change of $X$ is slow compared with the characteristic timescale of the motion of the individual oscillators, i.e. the variation of $X$ is assumed to be adiabatic. The dissipation is characterised by calculating the irreversible component of the rate of change of the energy of the gas of microscopic oscillators. By conservation of energy, this energy is lost from the motion of the observed degree of freedom.

$\dagger$ Permanent address: Department of Physics and Applied Physics, John Anderson Building, University of Strathclyde, Glasgow G4 0NG, UK. 


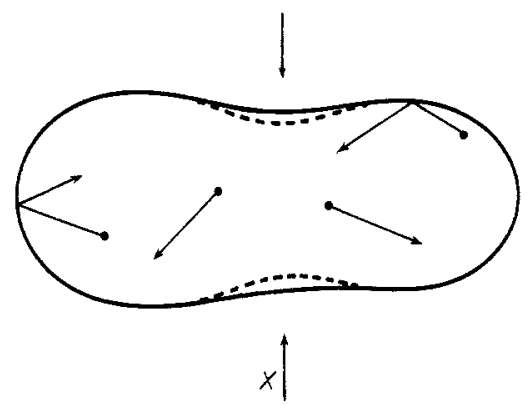

Figure 1. An example of the type of system being considered. A billiard is filled with a gas of non-interacting fermions. The shape of the billiard depends on a parameter $X$, which varies as a function of time, causing an irreversible increase in the energy of the gas. This results in the dynamics of the coordinate $X$ being dissipative.

An example of the type of system considered is illustrated in figure 1. A gas of particles is enclosed inside a wall, which can undergo a deformation described by a coordinate $X$. The motion of the gas particles is ballistic, i.e. the mean free path is assumed to be larger than the size of the enclosure. In the language of dynamical systems the gas is a system of billiards. It is well known that smooth-walled billiards can exhibit either regular or chaotic motion depending on the shape of the boundary (Berry 1976). Our object is to understand how this affects the ability of the gas to act as a dissipative medium.

One physical application of this billiard model is as an extension of the liquid-drop model of the nucleus to include the damping of the collective degrees of freedom by 'nuclear viscosity' (Hill and Wheeler 1952, Koonin and Randrup 1977, Blocki et al 1978). The billiard model is thought to be reasonable because two-body scattering of quasiparticles within the nuclear fluid is inhibited by the fact that the effective temperature is low. The dynamics of the collective coordinate $X$ describing the deformation of the nuclear fluid droplet are determined by inertial forces due to the bulk motion of the fluid when the drop is deformed, conservative forces involving surface tension and Coulombic repulsion, and a viscous force obtained by dividing the rate of dissipation by the velocity.

Another, closely related, physical application is to the absorption of electromagnetic radiation by small conducting particles. Here we assume that the particles are smaller than the bulk mean free path of the charge carriers, and that the frequency of the radiation is low compared with the frequency with which the particles collide with the wall. The coordinate $X$ is the electric or magnetic field of the radiation: instead of deforming the wall, we are now altering the dynamics within the enclosure.

To calculate the rate of dissipation we must estimate the change in the energy of the particles when the Hamiltonian is changed adiabatically. Integrable and chaotic Hamiltonian systems behave very differently in this respect, and will be discussed separately. Both integrable and chaotic systems have adiabatic invariants, and for each type of system the energy can be expressed as a function of the adiabatic invariants, which are functions of the parameter $X$. Irreversible effects are therefore associated with corrections to the adiabatic invariants due to the finite rate of change of the parameter $X$. 


\section{Ergodic systems}

First we discuss the case of ergodic systems in detail. The adiabatic invariant $\mu$ is the phase space volume of the energy shell:

$$
\mu=\int \mathrm{d} \boldsymbol{q} \int \mathrm{d} p \vartheta(H(\boldsymbol{q}, \boldsymbol{p} ; X)-E(X))
$$

(for a derivation, see Ott (1979)). The constancy of $\mu$ determines the dependence of the energy of an oscillator $E$ on the parameter $X$. This relationship therefore gives a contribution to the potential energy function for the coordinate $X$ due to its coupling to the $N$ oscillators. The leading-order term in the corrections to the adiabatic invariant has been calculated by Ott (1979), who showed that the differences $\Delta E$ between the actual energies of the particles and the energy predicted by the adiabatic invariant (1) increase diffusively

$$
\left\langle\Delta E^{2}\right\rangle=2 D t
$$

where the diffusion constant is proportional to the integral over time of the correlation function of the generalised force $\partial H / \partial X$ :

$$
\begin{gathered}
D=\frac{\dot{X}^{2}}{2 \Omega} \int_{-\infty}^{\infty} \mathrm{d} t C(E, t) \\
C(E, t)=\int \mathrm{d} \boldsymbol{q} \int \mathrm{d} \boldsymbol{p}\left(\frac{\partial H}{\partial X}(\boldsymbol{q}, \boldsymbol{p})-\left\langle\frac{\partial H}{\partial X}\right\rangle\right) \\
\times\left(\frac{\partial H}{\partial X}(\boldsymbol{Q}(t), \boldsymbol{P}(t))-\left\langle\frac{\partial H}{\partial X}\right\rangle\right) \delta(H(\boldsymbol{q}, \boldsymbol{p})-E) .
\end{gathered}
$$

In equation (4), $Q(t)$ is shorthand for the coordinate reached by evolving Hamilton's equations for time $t$ starting at $(q, p)$, i.e. $Q(t)=Q(q, p, t)$, and $\Omega$ is the weight of the energy shell at energy $E$ :

$$
\Omega(E)=\int \mathrm{d} \boldsymbol{q} \int \mathrm{d} p \delta(H(\boldsymbol{q}, \boldsymbol{p}, X)-E) .
$$

The diffusion of particle energies can be related to the irreversible component of the power supplied to the oscillators. The gas of oscillators is characterised by their phase-space density, $\rho(\boldsymbol{q}, \boldsymbol{p}, t)$. Initially, $\rho$ is assumed to be a function $f(E)$ of the energy $E=H(\boldsymbol{q}, \boldsymbol{p}, X)$. Although the time-evolution of $\rho$ is extremely complex, a coarse-grained average of $\rho$ depends only on the energy at any given time, and this average $f(E, t)$ satisfies the diffusion equation with drift

$$
\frac{\partial(\Omega f)}{\partial t}+\dot{X} \frac{\partial}{\partial E}\left(\frac{\mathrm{d} E}{\mathrm{~d} X} \Omega f\right)=\frac{\partial}{\partial E}\left(D \Omega \frac{\partial f}{\partial E}\right)
$$

where $D$ is the diffusion coefficient given by (2) and $\mathrm{d} E / \mathrm{d} X$ is the rate of change of the energy of a particle implied by the adiabatic invariant (1). The energy of the system of oscillators is given by

$$
E_{\mathrm{T}}=\int_{-\infty}^{\infty} \mathrm{d} E \Omega(E, X) f(E, t) E
$$

so that the rate of change of the energy of the system is

$$
\frac{\mathrm{d} E_{\mathrm{T}}}{\mathrm{d} t}=\dot{X} \int_{-\infty}^{\infty} \mathrm{d} E f \Omega \frac{\mathrm{d} E}{\mathrm{~d} X}-\int_{-\infty}^{\infty} \mathrm{d} E D \Omega \frac{\partial f}{\partial E} .
$$


The first term in this expression represents a reversible change in the energy of the system, and the second term represents the irreversible dissipation of energy into the bath of oscillators. Note that this dissipation is viscous or ohmic (force $\propto$ velocity), because the diffusion coefficient $D$ is proportional to the square of the velocity.

If the system of particles which act as the dissipative medium is a gas of fermions with an effective temperature which is low compared to the fermi temperature, then the phase space density $f(E)$ decreases rapidly from $1 / h^{d}$ to zero in the neighbourhood of the fermi energy $E_{\mathrm{F}}$ (where $h$ is Planck's constant and $d$ the number of degrees of freedom of each individual oscillator). In this case the rate of dissipation reduces to the simple expression

$$
\left(\frac{\mathrm{d} E_{\mathrm{T}}}{\mathrm{d} t}\right)_{\text {irrev }}=D_{\mathrm{F}} \Omega_{\mathrm{F}} / h^{d}
$$

where the subscripts $F$ indicate that quantities are evaluated at the Fermi energy. The physical interpretation of this result is that the diffusion of the distribution function $f(E, t)$ causes the occupation probability of states above the Fermi energy to be increased at the expense of states below $E_{\mathrm{F}}$, resulting in an irreversible increase in the energy of the Fermi gas. It may be thought that if the particles are fermions, the Pauli exclusion principle would inhibit the diffusion of the particle energies. This does not happen, however; it is simple to show that the evolution of a system of $N$ non-interacting fermions is given exactly by a Slater determinant of $N$ independently evolving onefermion wavefunctions, so that the Pauli principle cannot inhibit the diffusion of particle energies.

The appendix describes the results of numerical simulations which verify the relationship (9) between the diffusion of energy and the rate of dissipation.

\section{Integrable systems}

If the motion of the particles forming the dissipative medium is integrable, with motion confined to tori in phase space (Berry 1976), a different form of the adiabatic theorem is applicable. In the case of integrable motion with $d$ degrees of freedom the adiabatic invariants are the actions

$$
I_{\gamma}=\oint_{C_{\gamma}} p \cdot \mathrm{d} \boldsymbol{q}
$$

where the $C_{\gamma}$ are the $d$ irreducible circuits around the torus, and the energy can be expressed as a function of these adiabatic invariants: $E=H(I ; X)$. Dissipation must therefore be associated with corrections to the adiabatic invariants.

These corrections are much smaller than in the case of ergodic motion: for a generic perturbation of a one-freedom (necessarily integrable) system the irreversible component of the corrections to the adiabatic invariant (10) vanishes faster than any power of the velocity (Lennard 1959), provided $X(t)$ is an analytic function, and that the trajectory does not cross a separatrix as $X$ varies (Hannay 1986). The irreversible corrections in fact usually vanish exponentially

$$
\Delta I \simeq B \exp (-A / \dot{X})
$$

where $A$ is independent of $\dot{X}$, and $B$ may have a power-law dependence on $\dot{X}$. 
For integrable systems with more than one degree of freedom the accuracy of the adiabatic invariants is a delicate matter. If the system is integrable by separation of variables in the same coordinates system for all $X$, then (11) remains valid. In the general case of a one-parameter family of integrable systems, the adiabatic invariants are susceptible to irreversible changes if a particle is on a resonant trajectory. When there are no separatrices, however, the irreversible rate of change in the energy of the gas still vanishes more rapidly than $\dot{X}^{2}$ as $\dot{X} \rightarrow 0$.

Integrable systems are a special case, but many systems are quasi-integrable, with a mixture of regular motion, with trajectories on KAM tori, and regions where the motion is chaotic (see, e.g., Berry 1976). It is clear that in these cases there will be some ohmic dissipation associated with the chaotic regions.

The appendix describes the results of numerical experiments which show that the irreversible changes in energy are much smaller in integrable or quasi-integrable systems than in a comparable ergodic system, in the adiabatic limit.

\section{Possible experiments}

Now let us consider the possibilities for an experimental realisation of the effect of the classical dynamics on the rate of dissipation. A test involving the application to the problem of nuclear dissipation would be very difficult, because of the complexity of the system and the difficulty of controlling the parameters of the experiment. A much more straightforward application of the model may be to the absorption of low-frequency electromagnetic radiation by electrons confined within small conducting particles: the electric or magnetic field of the radiation plays the role of the slowly varying parameter $X$.

To perform experiments relevant to this model several criteria must be met. In order for the dynamics to correspond to that of a classical billiard, the bulk mean free path should be much greater than the size of the particles, and the surface should be smooth enough to give specular reflection (a criterion for this is that the surface roughness be much less than the inverse of the Fermi wavevector). In order for the adiabatic theory to apply, the time taken for an electron travelling at the Fermi velocity to cross the particle should be much less than the period of the radiation. Because the absorption increases as the square of the frequency, its detection is easier at high frequencies, which implies that the particles should be very small if the adiabatic condition is also to be satisfied. It must also be possible to prepare particles with both chaotic and integrable dynamics which are comparable in all other respects.

It may be possible to satisfy the above criteria in the following experiment: a very fine metallic powder is embedded in a non-conducting inert substrate, with a lower melting point than the metal. The particles in the powder would have irregular shapes, so that the motion of electrons within them would be chaotic. After measuring the absorption of the sample, it would be heated to melt the metallic particles, which would re-solidify as nearly spherical crystallites. Because the sphere is an integrable billiard, the absorption of the sample should then be greatly reduced, and scattering from impurities will be the dominant mechanism of dissipation, rather than scattering off the walls. The absorption coefficient would be decreased by (approximately) the ratio of the size of the particle to the bulk mean free path.

Bismuth may be a suitable substance for performing this experiment: it is a semi-metal, with a low carrier density $\left(10^{-5} /\right.$ atom), so that the Fermi energies of the 
electrons and holes are small (around $20 \mathrm{MeV}$, four pockets), with nearly ellipsoidal Fermi surfaces (Lerner 1962). The highest effective mass is around $m^{*}=1.2$ and the lowest is around $m^{*}=0.005$ (in units of the bare electron mass). These values lead to a smallest Fermi wavelength of approximately $75 \AA$, implying that surface irregularities can be many atomic diameters before they have any effect on the dynamics of the charge carriers. The smallest Fermi velocity is approximately $8 \times 10^{4} \mathrm{~m} \mathrm{~s}^{-1}$, implying that if the experiment were done with particles of size $10^{-7} \mathrm{~m}$, at a frequency of $10 \mathrm{GHz}$, the adiabatic criterion would be satisfied. This particle size is smaller than the bulk mean free path in Bismuth (approximately $10^{-6} \mathrm{~m}$, as estimated from the resistivity of $3.5 \times 10^{-7} \Omega \mathrm{m}$ at $77 \mathrm{~K}$ ). Because the Fermi surfaces are ellipsoids, the dynamics of the carriers in the spherical particles are equivalent to ellipsoidal rather than spherical billiards, but this makes no difference to the prediction because ellipsoidal billiards also have integrable dynamics.

Another possibility for an experimental system would be to use lithographic techniques to divide a surface holding a two-dimensional electron gas into regions with a precisely controlled shape, corresponding to billiards with known dynamics. The Fermi wavelength in these systems can also be very large, so that the effects of surface roughness can be suppressed.

\section{Discussion}

An expression for the rate of dissipation which is essentially equivalent in form to (8) or (9) can also be derived using classical linear response theory (see, e.g., Koonin and Randrup 1977). The advantages of the approach described in this paper are that it is not confined to small perturbations of the Hamiltonian, and that it shows clearly that the rate of dissipation is much lower if the classical motion of the oscillators is integrable.

The crucial difference between the problem considered here and the damping by harmonic oscillators considered by Caldeira and Leggett (1981) is that they consider a bath of harmonic oscillators with a continuous spectrum of frequencies: there are low-frequency oscillators which are not being driven adiabatically, and it is these which are responsible for the damping in their model.

All of the considerations above are based on a semiclassical model, and it remains to consider whether quantum mechanical effects will be significant. The model assumes that semiclassical considerations are valid for the static problem, and that the variation of the parameter $X$ is not so slow that the quantum adiabatic theorem (Bohm 1951) applies, rather than the classical one. Semiclassical models give a good description of the static problem if the Fermi wavelength is small compared with the size of the system. In the quantum adiabatic regime, Landau-Zener transitions at values of $X$ where pairs of energy levels become nearly degenerate (termed 'avoided crossings') are the only potential mechanism for irreversibility (Wilkinson 1988). The difference between the response of ergodic and integrable systems persists into the quantum adiabatic regime: in an integrable or quasi-integrable system, the gaps in the avoided crossings between energy levels are exponentially small (Wilkinson 1987). This implies that the probability of making a Landau-Zener transition is almost exactly unity, so that in these systems the transitions are almost exactly reversible on reversing the change in $X$.

In conclusion, this paper has given a simple expression for the rate of dissipation by a system of slowly driven identical oscillators with ergodic classical motion (9), and has pointed out that the rate of dissipation is drastically reduced if the classical 
motion is integrable. This model is relevant to the damping of collective motions in the atomic nucleus, and the absorption of low-frequency electromagnetic radiation by very small conducting particles. The parameters of the latter system can be controlled easily, and an experiment has been proposed which might provide a demonstration of the different rate of absorption for integrable and chaotic motion.

\section{Acknowledgment}

I am grateful to Dr W J Swiatecki and his colleagues at the Lawrence Berkeley Laboratory for their hospitality during my visit, which was paid for by US Department of Energy funds.

\section{Appendix}

This appendix describes some numerical experiments which verify the connection between dissipation and the diffusion of particle energies for an ergodic system, and which illustrate the dramatic difference between the chaotic and the integrable cases.

I considered three different Hamiltonians:

$$
\begin{aligned}
& H_{n}=\frac{1}{2}\left(p_{x}^{2}+p_{y}^{2}\right)+V_{n}(x, y, X) \\
& V_{1}=x^{2} y^{2}+\frac{1}{20}(1+X)\left(x^{2}+y^{2}\right) \\
& V_{2}=\frac{1}{2}\left(x^{2}+y^{2}\right)+\frac{1}{20}(1+X) x^{2} y^{2} \\
& V_{3}=x^{4}+6 x^{2} y^{2}+8 y^{4}+(1+X)\left(x^{2}+4 y^{2}\right) .
\end{aligned}
$$

The Hamiltonian $H_{1}$ is almost entirely chaotic at energy $E_{\mathrm{F}}=1$, for $X$ between 0 and 1 , whereas $\mathrm{H}_{2}$ is quasi-integrable (most trajectories are on tori) at $E_{\mathrm{F}}=1$. The Hamiltonian $\mathrm{H}_{3}$ is exactly integrable for all values of $X$ (Hietarinta 1984).

In the simulations the parameter $X$ was increased from 0 to 1 , and then reversed, with the time dependence

$$
X(t)=\exp \left(-t^{2} / 2 t_{\mathrm{s}}^{2}\right)
$$

For the chaotic Hamiltonian $H_{1}$, I computed the change $\Delta E_{\mathrm{T}}$ in the total energy of $N=2 \times 10^{4}$ particles numerically for various values of the switching time $t_{\mathrm{s}}$, with the initial phase points distributed uniformly inside the energy shell $E_{\mathrm{F}}=1$. Similar simulations were performed to evaluate $\left\langle\Delta E^{2}\right\rangle_{F}$, the mean value of the square of the changes in the single-particle energies, for initial conditions uniformly distributed on the energy shell at $E_{\mathrm{F}}=1$, averaging over 100 or more trajectories in each case. The results are summarised in table 1 . The weight of the energy shell, $\Omega$, and its volume $\mu$, were evaluated by Monte Carlo integration, using $10^{4}$ points: the values are listed in table 2 .

For a chaotic system, the increase in the total energy of the system is

$$
\Delta E_{\mathrm{T}}=N \Omega\left\langle\Delta E^{2}\right\rangle_{\mathrm{F}} / 2 \mu \text {. }
$$

This result follows from integrating (9), using the fact that the density of phase space points is $N / \mu$ in this simulation. The values of $\Delta E_{\mathrm{T}}$ in table 1 agree with this theoretical prediction to within the expected statistical fluctuations, and with the prediction (see (A.4) below) that $\Delta E_{\mathrm{T}}$ is proportional to $1 / t_{\mathrm{s}}$, in the limit $t_{\mathrm{s}} \rightarrow \infty$. It was necessary to use a large number of particles to get satisfactory agreement with the theory for large 
Table 1. Statistics describing the irreversible changes in energy caused by varying a parameter $X$, for various values of the timescale $t_{\mathrm{s}}$ of $X(t)$. For an explanation see the appendix.

\begin{tabular}{|c|c|c|c|c|c|c|c|}
\hline \multirow[b]{2}{*}{$t_{\mathrm{s}}$} & \multicolumn{3}{|c|}{$H_{1}$} & \multicolumn{2}{|c|}{$\mathrm{H}_{2}$} & \multicolumn{2}{|c|}{$\mathrm{H}_{3}$} \\
\hline & $\Delta E_{\mathrm{T}}$ & $\left\langle\Delta E^{2}\right\rangle_{F}$ & $\left\langle\Delta E^{2}\right\rangle_{\mathrm{est}}$ & $\left\langle\Delta E^{2}\right\rangle_{\mathrm{F}}$ & $\left\langle\Delta E^{2}\right\rangle_{\mathrm{est}}$ & $\left\langle\Delta E^{2}\right\rangle_{F}$ & $\left\langle\Delta E^{2}\right\rangle_{\text {est }}$ \\
\hline$\frac{1}{2}$ & - & - & - & $1.5 \times 10^{-4}$ & $6.0 \times 10^{-4}$ & $1.0 \times 10^{-3}$ & 0.354 \\
\hline 1 & - & - & - & $1.9 \times 10^{-5}$ & $3.0 \times 10^{-4}$ & $3.3 \times 10^{-7}$ & 0.177 \\
\hline 2 & - & - & - & $1.2 \times 10^{-9}$ & $1.5 \times 10^{-4}$ & $2.0 \times 10^{-7}$ & 0.089 \\
\hline 4 & - & - & - & $1.0 \times 10^{-9}$ & $7.5 \times 10^{-5}$ & $5.8 \times 10^{-8}$ & 0.044 \\
\hline 10 & 90.0 & 0.00544 & 0.0643 & $3.2 \times 10^{-9}$ & $3.8 \times 10^{-5}$ & $1.2 \times 10^{-9}$ & 0.018 \\
\hline 20 & 76.6 & 0.00532 & 0.0322 & $1.2 \times 10^{-9}$ & $1.5 \times 10^{-5}$ & $4.6 \times 10^{-11}$ & 0.009 \\
\hline 40 & 60.9 & 0.00403 & 0.0161 & $5.3 \times 10^{-11}$ & $7.5 \times 10^{-6}$ & $1.5 \times 10^{-12}$ & $4.4 \times 10^{-3}$ \\
\hline 80 & 36.7 & 0.00239 & 0.0081 & $4.7 \times 10^{-13}$ & $3.8 \times 10^{-6}$ & $1.1 \times 10^{-13}$ & $2.2 \times 10^{-3}$ \\
\hline 160 & 26.1 & 0.00120 & 0.0040 & - & - & $4.5 \times 10^{-14}$ & $1.1 \times 10^{-3}$ \\
\hline 320 & - & 0.00066 & 0.0020 & - & - & - & - \\
\hline
\end{tabular}

Table 2. Some phase-space integrals required for analysis of the data of table 1. For an explanation see the appendix.

\begin{tabular}{lccc}
\hline & $H_{1}$ & $H_{2}$ & $H_{3}$ \\
\hline$\mu$ & 51.4 & 19.5 & 3.65 \\
$\Omega$ & 84.0 & 38.5 & 6.63 \\
$C_{0}$ & 6.75 & $5.48 \times 10^{-3}$ & 0.982 \\
$C_{0}^{\prime \prime}$ & -1.33 & $-1.52 \times 10^{-2}$ & -8.61 \\
\hline
\end{tabular}

values of $t_{\mathrm{s}}$, because the mean change in the energy of each particle (which scales as $t_{\mathrm{s}}^{-1}$ ) is small compared to its standard deviation (which scales as $t_{\mathrm{s}}^{-1 / 2}$ ).

In order to make a quantitative comparison between the ergodic and integrable systems, we must compare the values of $\left\langle\Delta E^{2}\right\rangle_{F}$ we observe for the integrable system with that expected for a comparable chaotic system (i.e. one with a similar correlation function). For a chaotic system the variance $\left\langle\Delta E^{2}\right\rangle_{\mathrm{F}}$ is proportional to the integral over time of the diffusion constant: using (3) and (4) we have

$$
\left\langle\Delta E^{2}\right\rangle_{\mathrm{F}}=2 \int D \mathrm{~d} t^{\prime}=\frac{C_{0} \tau}{\Omega} \int \dot{X}^{2} \mathrm{~d} t^{\prime}=\frac{C_{0} \tau}{\Omega t_{\mathrm{s}}} \sqrt{2 \pi}
$$

where $C_{0}$ and $\tau$ are measures of the correlation function and its characteristic decay time, defined by:

$$
C_{0}=C(E, 0)=\int \mathrm{d} q \mathrm{~d} p\left(\frac{\partial H}{\partial X}\right)^{2} \delta(E-H) \quad C_{0} \tau=\int_{-\infty}^{\infty} \mathrm{d} t C(E, t) .
$$

In the case of an integrable system, the integral in (A.5) which defines $\tau$ may not converge, so a different characteristic time will be used as the basis for a comparison between the integrable and chaotic systems: this timescale $\tau_{0}$ defined by

$$
\tau_{0}=\left(2 C_{0} /-C_{0}^{\prime \prime}\right)^{1 / 2}
$$


where $C_{0}^{\prime \prime}$ is the second time derivative of the correlation function at $t=0$ :

$$
C_{0}^{\prime \prime}=\left.\frac{\mathrm{d}^{2} C(E, t)}{\mathrm{d} t^{2}}\right|_{t=0}=\int \mathrm{d} \boldsymbol{q} \mathrm{d} \boldsymbol{p}\left(\frac{\partial H}{\partial X}\right)\left\{\left\{\frac{\partial H}{\partial X}, H\right\}, H\right\} \delta(E-H)
$$

( $\{A, B\}$ is the Poisson bracket of $A$ and $B$ ). Both $C_{0}$ and $C_{0}^{\prime \prime}$ were evaluated by Monte Carlo integration $\left(10^{4}\right.$ points), and their values are given in table 2 . The columns in table 1 headed $\left\langle\Delta E^{2}\right\rangle_{\text {est }}$ are estimates for $\left\langle\Delta E^{2}\right\rangle_{\mathrm{F}}$ obtained from (A.4), with $\tau$ replaced by $\tau_{0}$.

It can be seen that the estimates $\left\langle\Delta E^{2}\right\rangle_{\text {est }}$ are of the right order of magnitude for the ergodic system, but much too high for the systems with integrable or near-integrable motion, especially in the adiabatic limit where $t_{\mathrm{s}}$ is large. This confirms the prediction that the irreversible energy changes caused by adiabatic perturbations are much smaller if the motion is integrable.

\section{References}

Berry M V 1976 Regular and irregular motion Topics in Nonlinear Dynamics ed S Jorna (AIP Conference Proceedings 46) (New York: AIP)

Blocki J, Boneh Y, Nix J R, Randrup J, Robel M, Sierk A J and Swiaetecki W J 1978 Ann. Phys., NY 113 130

Bohm D 1951 Quantum Theory (New York: Prentice-Hall)

Caldeira A O and Leggett A J 1981 Phys. Rev. Lett. 46211

Hannay J H 1986 J. Phys. A: Math. Gen. 19 L1067-72

Hietarinta J 1984 J. Math. Phys. 25 1833-44

Hill D L and Wheeler J A 1952 Phys. Rev. 89 1102-45

Koonin S E and Randrup J 1977 Nucl. Phys. A 289 475-510

Lennard A 1959 Ann. Phys, NY 6261

Lerner L S 1962 Phys. Rev. 127 1480-92

Ott E 1979 Phys. Rev. Lett. 42 1628-31

Pines D and Nozieres P 1966 The Theory of Quantum Liquids I (Menlo Park, CA: Benjamin)

Wilkinson M 1987 J. Phys. A: Math. Gen. $20635-45$

- 1988 J. Phys. A: Math. Gen. 21 4021-37 